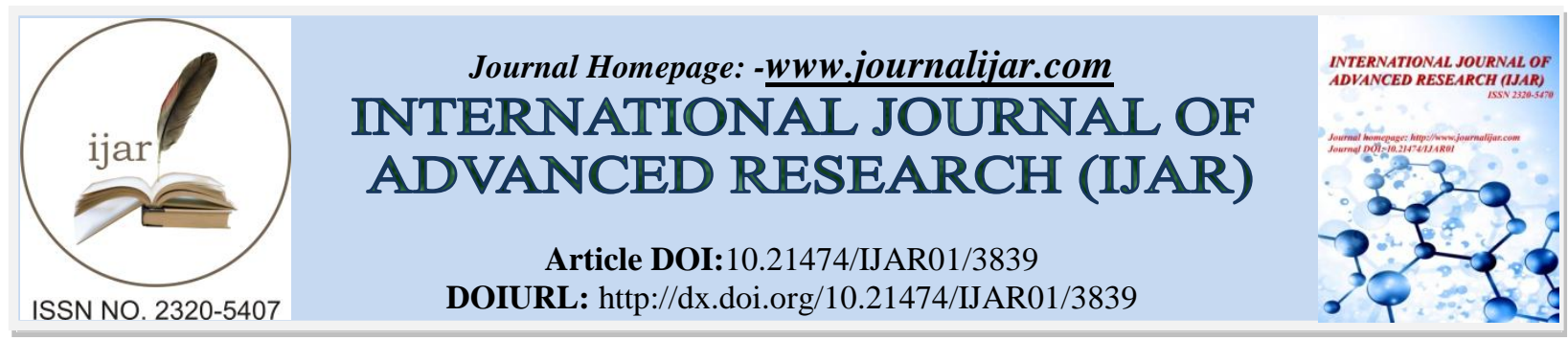

RESEARCH ARTICLE

\title{
DEVELOPMENT AND OPTIMIZATION OF A PHOTOVOLTAIC PUMPING IRRIGATION SYSTEM.
}

Khalid Assalaou ${ }^{1}$, Lahoussine Bouhouch ${ }^{2}$ and Lahoussine Elmahni ${ }^{3}$.

1. Department Of Physics, University Of Abdelmalek Essaâdi, Polydisciplinary Faculty, Larache, Morocco

2. ERTAIER, Ecole Supérieure De Technologie D'agadir (Esta), Bp 33/S, 80050, Agadir, Maroc

3. LMTI, Laboratoire De Metrologie Et Traitement De L'information, Faculte Des Sciences Agadir, Maroc

\section{Manuscript Info}

\section{Manuscript History}

Received: 01 February 2017

Final Accepted: 06 March 2017

Published: April 2017

Key words:-

Photovoltaic pumping station

Irrigation Arduino

\begin{abstract}
In the present study, we are interested in improving the development and optimization of an agricultural system based on photovoltaic (PV) pumping, through the programming of control and control cards. These boards, or modules of the Arduino type, will ensure better management of both electrical energy and water resources. The evaluation of the efficiency of these modules is carried out through a series of tests on a model prototype that we have designed. These tests show that in automated pumping, control boards allow not only the management and control of the operation of the pumping station for the whole year through the recording of the data but also the saving of water and electricity, in addition to the protection of the submerged pump.

As for automated irrigation, the control cards ensure a better use of water, depending on the specific needs of the plants and at a rate dictated automatically by the need of the soil.
\end{abstract}

Copy Right, IJAR, 2017,. All rights reserved.

\section{Introduction:-}

Au Maroc, les fermes sont assez souvent aménagées et se présentent sous forme de terrains découverts et / ou semés de serres, plus ou moins alimentées en eau à partir de puits munis de pompes immergées qui remplissent des bassins dotés à leur tour de pompes surfaciques. Ces pompes sont généralement alimentées par le réseau électrique public, ce qui occasionne malheureusement des dépenses budgétaires assez importantes, en électricité ou en butane ou encore en gasoil. Ces deux sources d'énergies sont nocives par-dessus le marché.

De telles fermes poussent à s'interroger sur les inconvénients de ces utilisations énergétiques, aussi bien que sur des alternatives à la lumière des nouvelles orientations universelles en matière d'énergies propres [1].

D'autres questions s'imposent, telles l'utilisation irrationnelle de l'eau dans la mesure où l'irrigation se fait sans soucis d'économie et sans dosage ni quantitatif ni temporel.

L'idée de recours au pompage PV surgit alors, comme moyen de réaliser des gains pécuniaires, tout en respectant l'environnement : plus de facture d'électricité pour le cultivateur et plus de pollution ni pour la faune ni pour la flore [2].Néanmoins, le débordement du bassin est toujours possible dans les systèmes de pompage si aucune protection n'est prévue pour l'arrêter. Cet effet en trainera alors des nuisances potentiellement importantes et graves; telles la création de zones humides propices à la présence d'insectes, la détérioration de la qualité environnementale du lieu, la dégradation des fondations du support, etc. 
Ceci nous astreint à chercher en hâte et méticuleusement des modes de gestion et d'exploitation d'eau à même d'optimiser le fonctionnement des pompes, en vue d'améliorer le rapport entre les besoins en eau, la durée de pompage et l'intensité maximale du rayonnement solaire, tout en favorisant la qualité des vies faunique et florale.

Des initiatives d'économie d'électricité et d'eau ont certes précédé notre étude. Ainsi, en matière de rentabilité économique, le coût du $\mathrm{m}^{3}$ d'eau pompé par les systèmes de pompage à énergie solaire PV s'est avéré meilleur que celui à énergie fossile. C'est qu'il s'agit d'un système qui se passe de tout carburant pour fonctionner et qui ne requiert guère d'entretien régulier [3], [4], [5].Toujours en matière d'économie, lorsqu'un bon dimensionnement du système de pompage PV est assuré, alors le fonctionnement de ce système se trouve optimisé [6], [7], [8]. Par ailleurs, ce système est reconnu comme plus fiable, grâce à une bonne optimisation et au contrôle du système d'irrigation [9], [10],[11], [12].

En fait, tout en garantissant l'autonomie, l'intelligence et des économies financières, notre présente étude s'intéresse à deux aspects : la conception et la réalisation d'un système automatisé, assurant d'abord le pompage PV et la gestion de l'irrigation d'un champ.

En matière d'automatisation de pompage PV, nous avons pressenti la nécessité de mettre en œuvre un mécanisme de contrôle et de fonctionnement régulier de la station de pompage.

Quant à la gestion de l'irrigation, nous devons trouver un moyen de dosage en eau selon les besoins spécifiques des plantes et selon une cadence dictée automatiquement par le besoin du sol.

Tel, est donc l'ambition de notre projet qui sera développé en trois parties à travers cet article : une partie pour le système automatisé de pompage $\mathrm{PV}$, une autre pour le système automatisé d'irrigation et une dernière que nous consacrerons à la discussion des résultats obtenus.

\section{Le Système De Pompage PV :-}

Au niveau de la figure 1, nous présentons le système global d'irrigation par pompage PV qui sera traité ci-après. En fait, il s'agit d'un système assurant : l'automatisation du remplissage du bassin, la protection de la pompe immergée, et la mesure instantanée du débit et de la consommation en eau. A ceci s'ajoute la visualisation, sur un afficheur LCD, des différents états de la station de pompage. En plus, les débits journaliers et la quantité consommée en eau sont affichés et stockés sur un ordinateur.

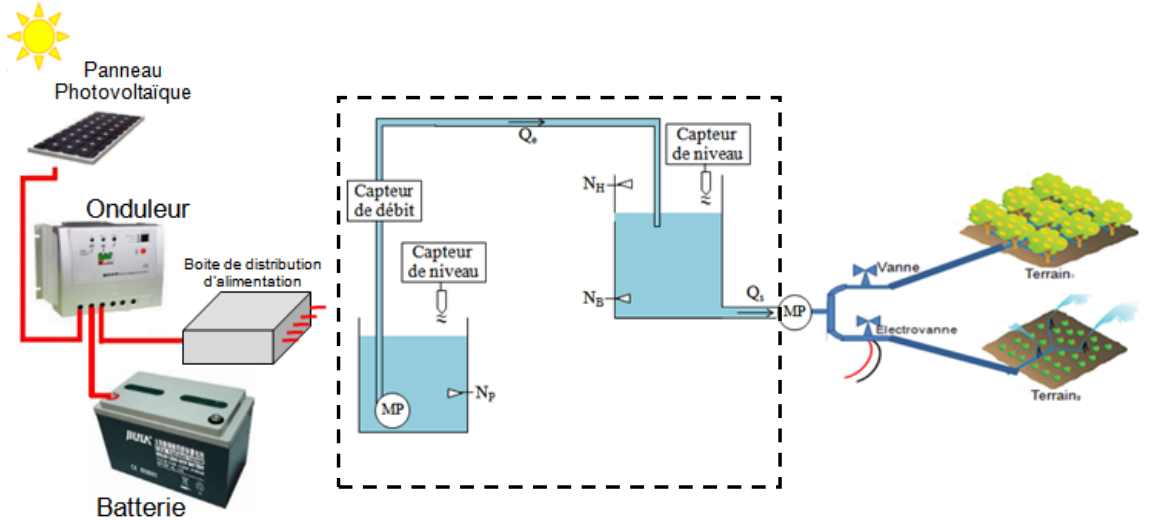

Fig. 1. Système automatique de pompage (encadré pointillé)et d'irrigation aliméntés par PV

\section{Description du système de pompage PV:-}

La maquette du prototype de pompe PV est destinée à étudier les problèmes que l'on peut rencontrer lors de l'installation d'un tel système dans un pays en voie de développement. Cette maquette (Figure2) reposant sur un bâti réalisé en acier, est composée des éléments suivants :

- Un récipient en plastique situé au niveau du plancher émulant un "puits",

- Une pompe d'essuie-glace en guise de "motopompe",

- Un récipient sous forme de "bassin" faisant office de réservoir,

- Des sources d'alimentation composée du couplage entre panneau PV, et onduleur,

- Un système de commande et de contrôle composé de cartes de type Arduino UNO programmées, munis de divers capteurs ainsi que de dispositifs de visualisation et d'alarme sonore. 


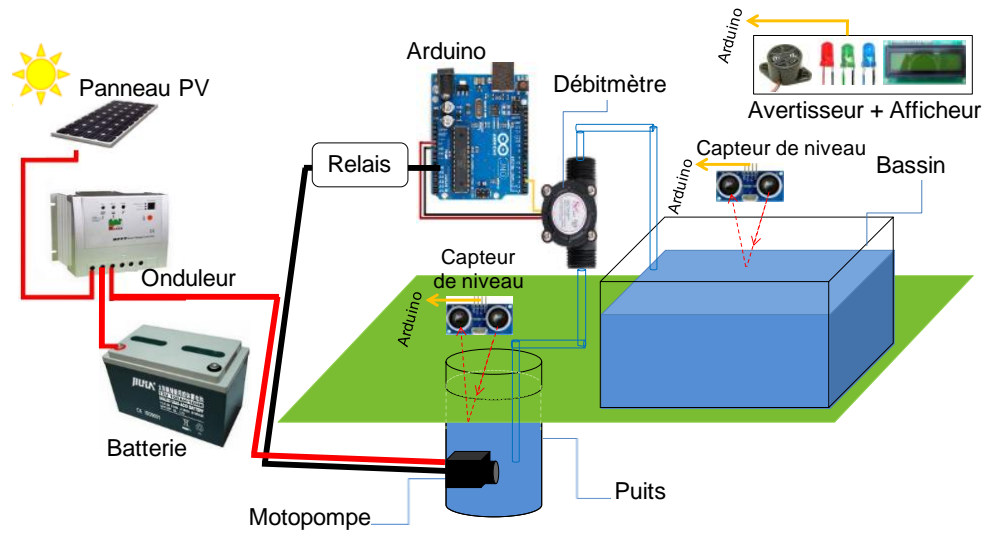

Fig. 2. Schéma de principe de la station de pompage PV

La conception de cette maquette est effectuée en utilisant le logiciel CATIA pour la facilité qu'il procure quant au passage à sa réalisation.

\section{La Carte Electronique De Commande Et De Contrôle:-}

Le schéma de principe de la partie électronique est représenté sur la Figure 3. L'alimentation de cette partie, comme d'ailleurs la "motopompe", est assurée par un panneau PV ou une batterie au plomb. Ce schéma de principe est constitué essentiellement des parties suivantes :

La figure 3illustre le schéma de principe constitué de plusieurs parties : la carte de commande de la station de pompage sous test, la carte renfermant une unité de mesure, une autre carte de traitement et une troisième constitué d'actionneurs. L'ensemble de ces cartes, comme d'ailleurs les "motopompes" et les électrovannes, sont alimentés par le bloc alimentation qui comprend un panneau PV, et un onduleur. Plus précisément, l'alimentation des cartes est assurée par une alimentation stabilisée que nous avons conçue, qui elle, est alimentée par l'onduleur via le panneau PV.

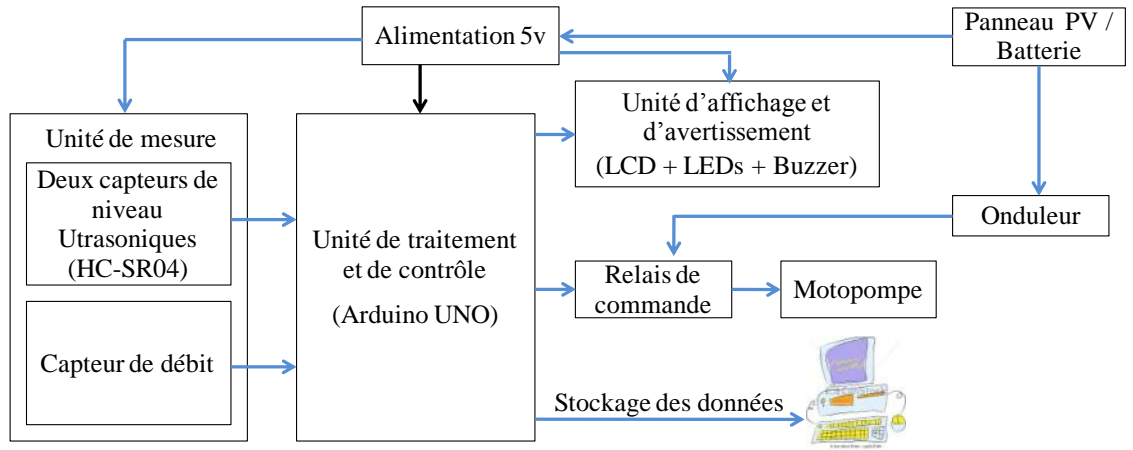

Fig. 3. Schéma de principe de la station de pompage

L'unité de mesure est composée de deux capteurs de position ultrasonores de type HC-SR04 [13], et d'un capteur de débit de type YF-DN50 [14].Les capteurs de positon servent à mesurer les distances entre la surface de l'eau et les positions des capteurs, lesquels sont positionnés l'un en haut du "puits" et l'autre en haut du "bassin". Quant au capteur de débit, il permet la mesure et le suivi des débits journaliers et des quantités consommées en eau.

L'unité de traitement, bâtis autour du microcontrôleur de la carte Arduino Uno, est programmée pour assurer l'automatisation de la station de pompage. Cette unité capte les informations délivrées par les différents capteurs et commande en conséquence des actionneurs.

Ces derniers se chargent du démarrage ou l'arrêt automatique de la pompe. L'affichage et/ou l'avertissement est assuré par l'unité d'afficheurs LCD, LEDs et BUZZER. 
En plus, un ordinateur relié à ce système par la liaison série USB, se charge d'archiver les débits journaliers ainsi que les quantités consommées en eau.

La Figure4 présente le schéma électrique de la carte de commande de la station de pompage. Cette carte a été utilisée pour effectuer diverses simulations sous le logiciel ISIS [16] avant de procéder aux tests pratiques de la réalisation.

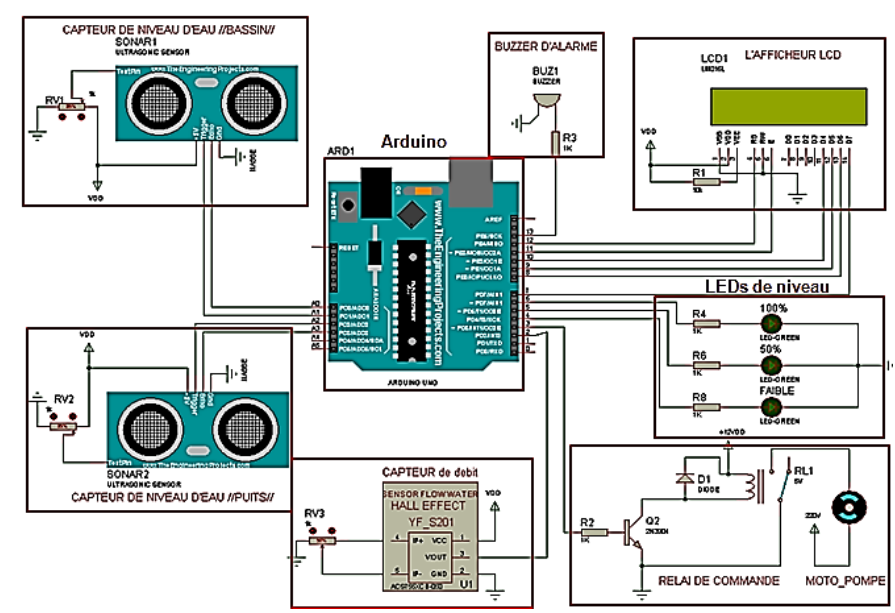

Fig. 4. Schéma électriquede simulation de la carte de commande de la station de pompage

\section{La carte Arduino et programme implémenté:-}

Il s'agit en fait d'une plateforme open-source, nommée Arduino UNO, bâtie autour d'un microcontrôleur 8 bits D'ATEML, de référence ATMega328, avec des composants complémentaires facilitant la programmation et l'interfaçage avec d'autres circuits électroniques (Figure 5)[15].

Ce microcontrôleur peut être programmé à travers l'IDE Arduino qui permet d'éditer, de compiler et transférer un programme vers sa mémoire. Il suffit simplement de connecter la carte mère Arduino à un ordinateur à l'aide d'un câble USB/Série.

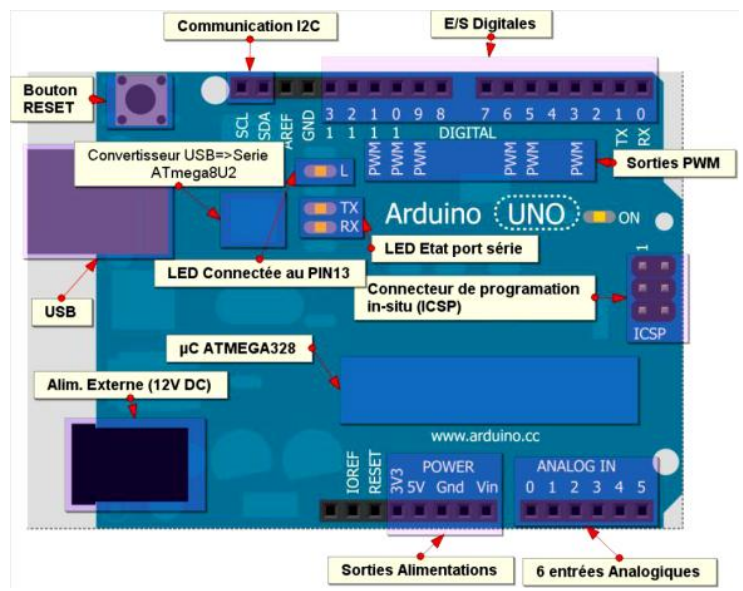

Fig. 5. Structure externe de la carte Arduino Uno

Le programme développé et implémenté est représenté par l'organigramme de la figure 6-a. La figure 6-b montre l'emplacement de deux capteurs ultrasonores, l'un pour le bassin $\left(C_{B}\right)$ et l'autre pour le puits $(C p)$. Ces derniers effectuent la mesure des diverses distances, telles que :

- $D p$ : Distance séparant le niveau d'eau du puits et celui du capteur $C p$.

- $D_{P r o}$ : Distance de protection entre le capteur $C p$ et la motopompe (MP).

- $D_{B}$ : Distance séparant le niveau d'eau du bassin et celui du capteur $C_{B}$.

- $D_{\text {Min }}$ : Distance entre le capteur $C_{B}$ et le niveau haut $N_{\text {Haut }}$ du bassin.

- $D_{M a x}$ : Distance entre le capteur $C_{B}$ et le niveau bas $N_{B a s}$ du bassin en amont du remplissage du bassin. 


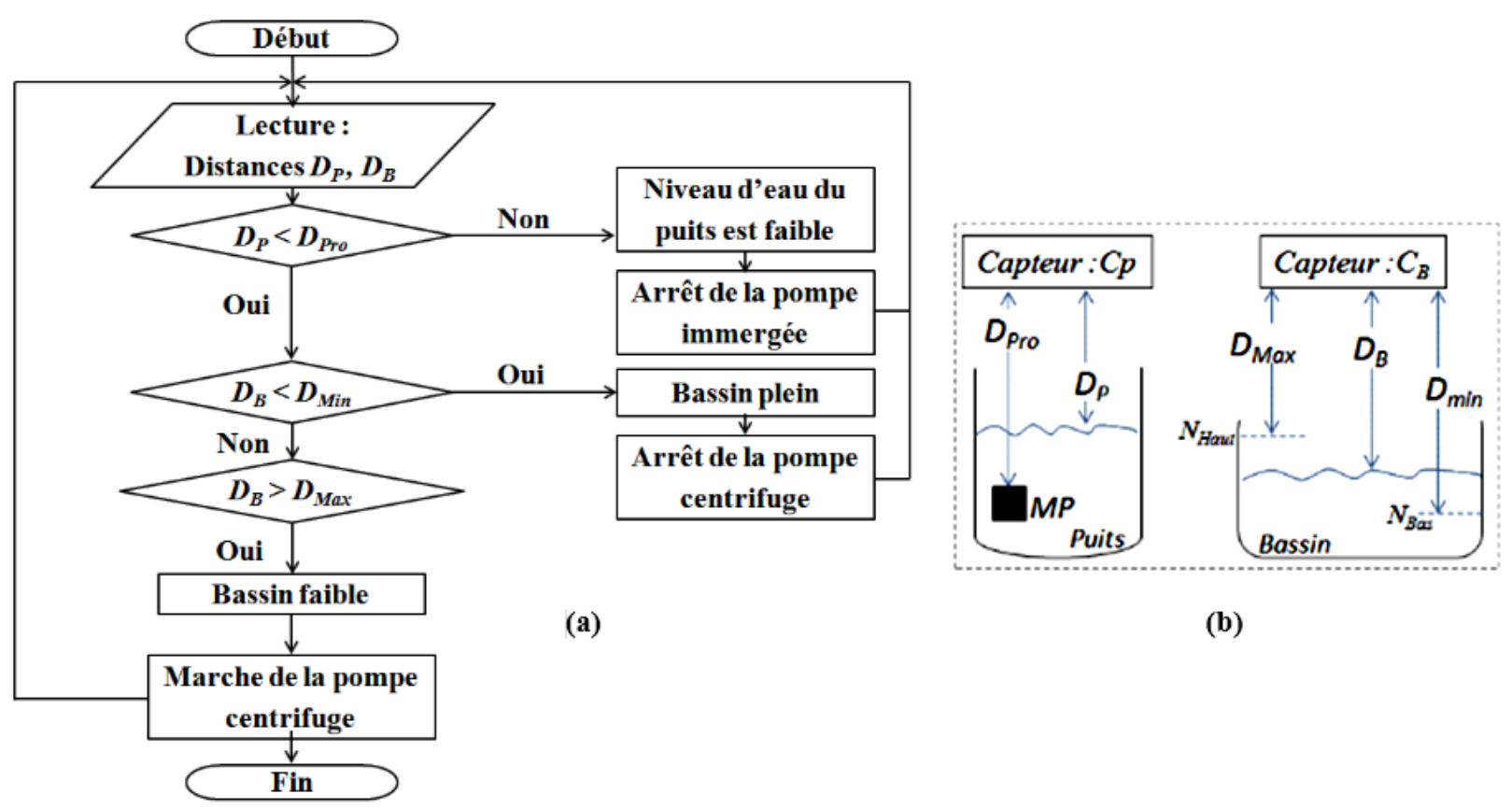

Fig. 6. (a) Organigramme décrivant le principe de commandede la station de pompage PV avec

(b) les divers paramètres

En fait le capteur $C_{B}$ détecte le niveau d'eau dans le "bassin" et par conséquent, envoie un signal à la carte Arduino. Ce signal correspond à une tension analogique équivalente à la distance entre ce capteur et le niveau d'eau dans le "bassin". Le programme développé pour ce capteur fonctionne de telle sorte que, si le niveau d'eau dans le bassin est très bas, soit $N_{\text {Bas }}$, alors la carte Arduino donne l'ordre d'amorcer la "motopompe" pour aspirer l'eau depuis le "puits" et la refouler dans le "bassin", jusqu'à ce que le niveau d'eau $N_{\text {Haut }}$ désiré soit atteint. Dans ce cas, la carte Arduino ordonne l'arrêt de la "motopompe".

Monté à l'extrémité haute du "puits", le second capteur ultrasonore informe la carte Arduino du niveau d'eau dans le "puits". Si ce niveau est inférieur à $N_{P}$, celui de la pompe immergée, alors la "motopompe" sera mise hors circuit quel que soit l'état de $N_{\text {Bas }}$ et $N_{\text {Haut }}$ et ce, jusqu'à la régénération naturelle de l'eau dans le puits. Etant donné que la motopompe est immergée, alors sa protection contre le fonctionnement à sec doit être prise en compte en amont du remplissage du bassin et ce, par comparaison des signaux $\left(V_{D p}\right.$ et $\left.V_{D B}\right)$ émis par le capteur de niveau $C p$, en procédant au calcul de leurs différences et en envoyant des impulsions à la motopompe entrainant son déclenchement ou son arrêt.

\section{Le système d'irrigation:-}

Compte tenu des imperfections qualifiant les pratiques traditionnelles d'arrosage, où la consommation en eau s'avère irrationnelle, du fait que les programmateurs en vigueur fonctionnent selon des horaires fixes préalablement enregistrés et partant, insoucieux des différences contextuelles inhérentes à l'humidité, la température, la ventilation et l'ensoleillement, nous envisageons développer une nouvelle modalité d'arrosage. Elle tiendra compte de la luminosité et de l'humidité du sol à l'aide des capteurs, permettant ainsi que la détection du besoin des plantes en eau et par conséquent la gestion de l'irrigation selon le temps et la durée d'arrosage ainsi que le débit en eau à mettre en œuvre. Les évènements détectés et les décisions prises sont visualisés sur un afficheur de type LCD.

\section{Description du système d'irrigation :-}

Techniquement, comme l'illustre la figure7, notre système d'irrigation est constitué essentiellement d'un bassin relié à une pompe surfacique pilotée par un programmateur. Cette pompe automatique assure le refoulement de l'eau vers les deux champs à arroser via une rampe comportant deux électrovannes. Ces deux champs sont munis de capteurs d'humidité du sol et d'un capteur de luminosité. Ces capteurs renseignent le programmateur qui déclenche l'ouverture ou la fermeture automatique des électrovannes. 


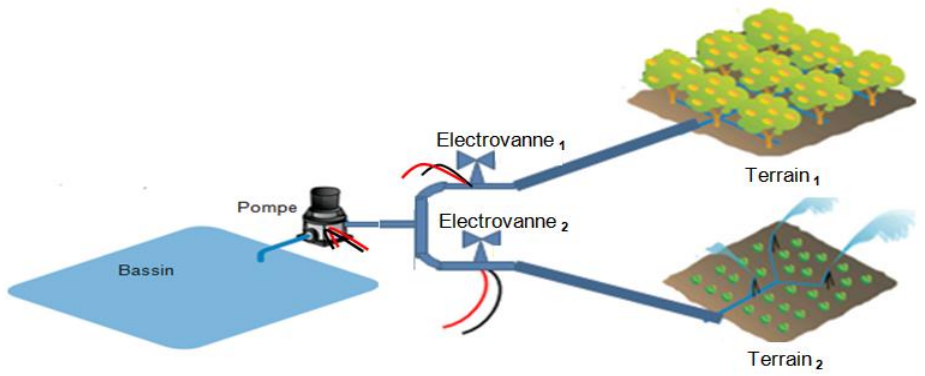

Fig. 7. Schéma de principe de l'irrigation

Cependant, parfois il y a des risques d'anomalie ou de panne de l'une des électrovannes. Dans ce cas, après le signalement lumineux (LED) ou sonore (Buzzer), nous faisons recours à une vanne manuelle montée en parallèle avec l'électrovanne. En outre, le Buzzer, connecté à un programmateur, réagit en cas de présence d'humidité dans le terrain relié à l'électrovanne inactive.

\section{Le schéma synoptique de la carte électronique:-}

Comme le montre la figure 8 , le schéma synoptique du système d'irrigation est constitué essentiellement de trois parties décrites ci-après. En plus du bloc d'alimentation qui comprend l'onduleur, le panneau PV et la batterie, ces parties sont:

$>$ Une carte renfermant une unité de mesure via des capteurs.

$>$ Une carte de traitement et de contrôle.

$>$ Un circuit renfermant les actionneurs munis d'indicateurs.

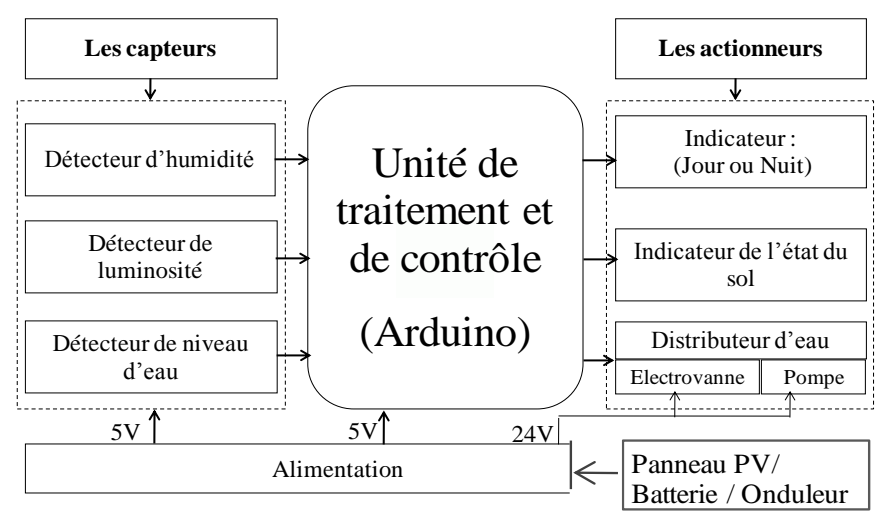

Fig. 8. Schéma synoptique du système d'irrigation

$>$ L'unité de mesure comprend plusieurs types de capteurs, à savoir des capteurs d'humidité, de luminosité et de niveau d'eau du bassin. Les premiers mesurent l'humidité du sol. Ceux de luminosité sont utilisés de manière à ce que la pompe ne soit déclenchée que pendant la journée. Quant à ceux de niveau, ils permettent de détecter la présence de l'eau dans le bassin avant le déclenchement de l'irrigation. Ces trois capteurs sont alimentés par un panneau PV.

$>$ L'unité de traitement se charge de capter les informations délivrées par les différents capteurs. Il s'agit du microcontrôleur logé dans la carte Arduino UNO, lequel microcontrôleur assure la gestion de l'irrigation.

$>$ Les actionneurs sont pilotés par la carte Arduino selon le programme préétabli. Il s'agit de la commande de la pompe surfacique et des électrovannes. Dans cette partie, sur un écran LCD, nous retrouvons également l'affichage de l'état de la pompe (On/Off) ainsi que celui du sol (Humide/Sec) avec également l'indication Jour/Nuit.

\section{Description du schéma Electronique:-}

La Figure 9 présente le schéma électrique de la carte de commande du prototype de la station de pompage. Cette carte a été simulée sous le logiciel ISIS. 


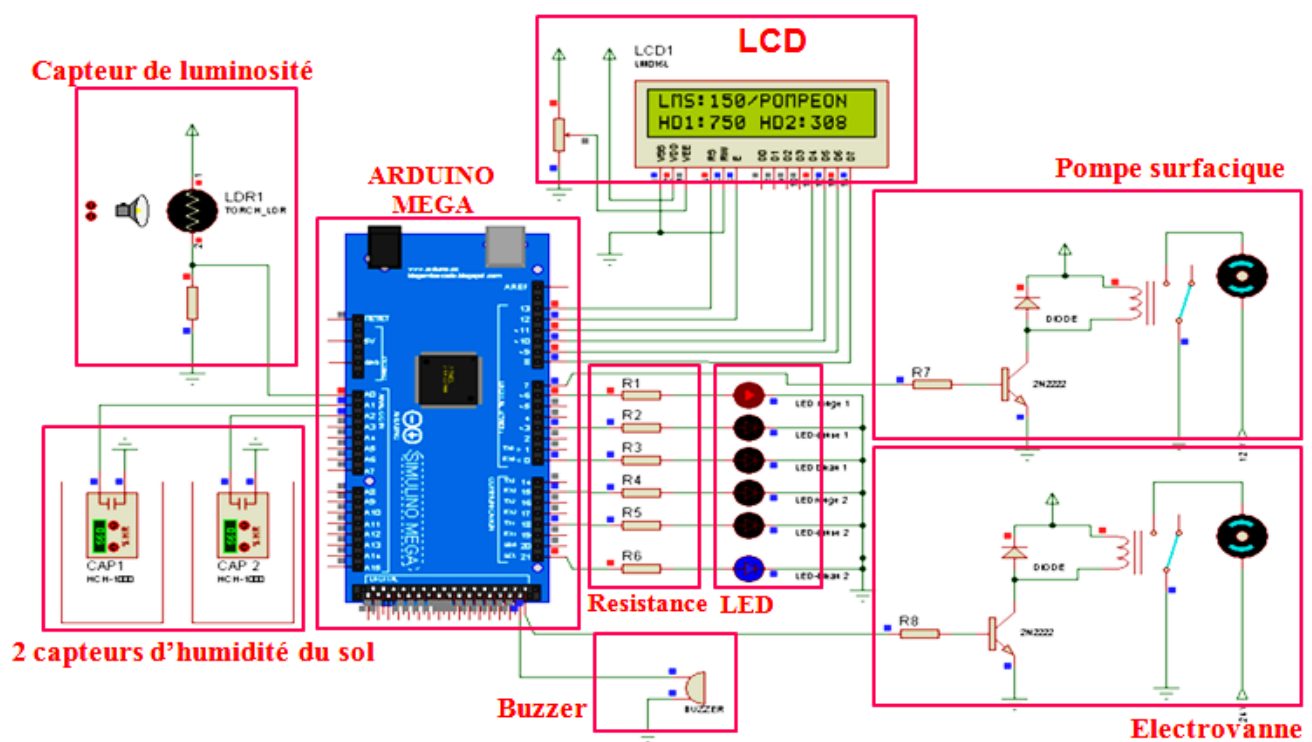

Fig. 9. Schéma électrique du système d'irrigation automatique

\section{Résultat et Discussion:-}

La figure 10 montre la maquette reflétant l'aboutissement des travaux mentionnés ci-dessus. Cette maquette a été soumise à des tests de fonctionnement qu'illustre le tableau 1 en tant que table de vérité applicable à deux champs dans les cas ordinaires.

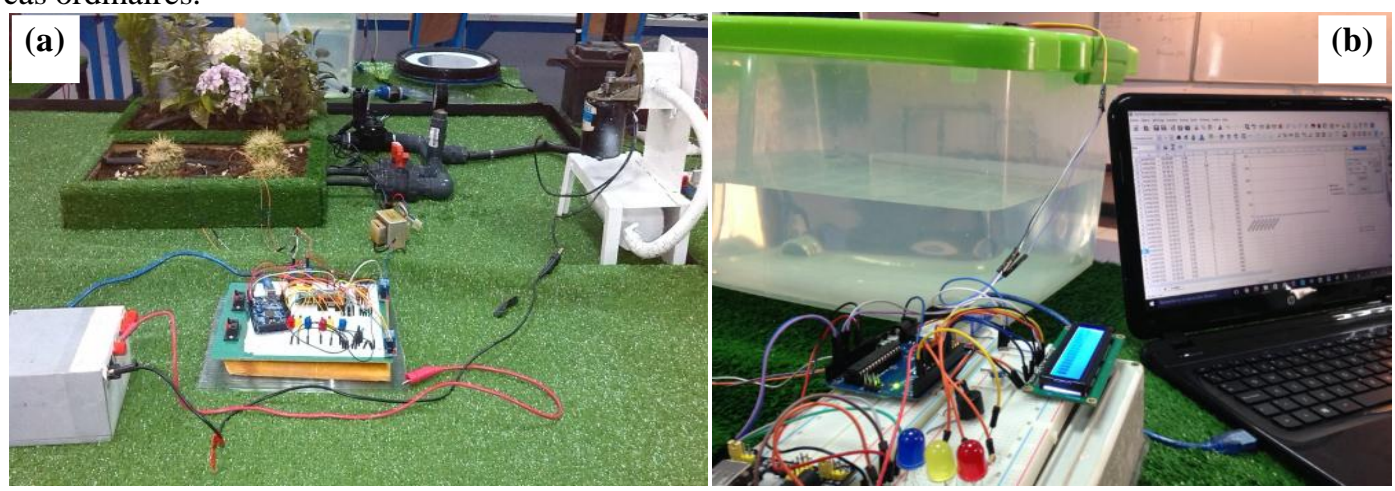

Fig. 10. Maquette de l'irrigation automatique (a) et de la station de pompage d'eau (b)

Tableau 1:- Table de vérité d'arrosage automatique ordinaire.

\begin{tabular}{|c|c|c|c|c|}
\hline \multirow[t]{2}{*}{$\operatorname{Cas}^{\circ}$} & \multicolumn{2}{|c|}{ Etat des Champs } & \multirow[t]{2}{*}{ Etat de l'Electrovanne $_{1}$} & \multirow[t]{2}{*}{ Etat de l'Electrovanne $_{2}$} \\
\hline & (1) & (2) & & \\
\hline 1 & Humide & Humide & OFF & OFF \\
\hline 2 & $\mathrm{Sec}$ & $\mathrm{Sec}$ & ON & ON \\
\hline 3 & Humide & $\mathrm{Sec}$ & OFF & ON \\
\hline 4 & $\mathrm{Sec}$ & Humide & OFF & OFF \\
\hline
\end{tabular}

Le nombre des champs à irriguer peut-être supérieur à deux dans cette table de vérité. Dans ce cas, pour $n$ champs donnés, les possibilités sont estimées à $2^{n}$.

En cas d'anomalie ou de panne de l'une des électrovannes, nous faisons recours à une vanne manuelle montée en parallèle avec l'électrovanne. En outre, le Buzzer, connecté à un programmateur, réagit en cas de présence d'humidité dans le terrain relié à l'électrovanne inactive. Ladite situation se présente comme le montre le tableau 2. 
Tableau 2:- Table de vérité de l'irrigation automatique en cas d'anomalie.

\begin{tabular}{|c|c|c|c|c|c|}
\hline \multirow{2}{*}{ Cas $\mathbf{N}^{\circ}$} & \multicolumn{2}{|c|}{ Etat des Champs } & \multirow{2}{*}{ Pompe } & Etat de l'Electrovanne & Etat du Buzzer \\
\cline { 2 - 3 } & $\mathbf{( 1 )}$ & $\mathbf{( 2 )}$ & & Champs(2) & Champs(1) \\
\hline 1 & Humide & Humide & OFF & OFF & OFF \\
\hline 2 & Sec & Sec & ON & ON & OFF \\
\hline 3 & Humide & Sec & ON & ON & ON \\
\hline 4 & Sec & Humide & ON & OFF & OFF \\
\hline
\end{tabular}

Dans ce cas d'anomalie, le tableau 3 présente les différents états de champs que signifient les LEDs. Ces dernières noue indique l'état de l'humidité du sol afin d'apporter la bonne quantité d'eau aux cultures, au bon moment.

Tableau 3:- Table de vérité de l'irrigation automatique.

\begin{tabular}{|c|c|c|c|c|}
\hline Etat du Champs & Humidité "H" & Résistivité "Res" & LED & Irrigation \\
\hline Sec & $H<25 \%$ & $>50 \%$ & Rouge & Oui \\
\hline Semi-Humide & $25 \%<H<50 \%$ & $25 \%<\operatorname{Res}<50 \%$ & Jaune & Non \\
\hline Humide & $H>50 \%$ & $<50 \%$ & Bleu & Non \\
\hline
\end{tabular}

\section{Remerciements:-}

Les auteurs tiennent à remercier les étudiants : Najoua Ben hamed, Jamal Eddib, Issam El Khlifi et Ahmed El Otmani pour leur contribution et aide précieuse concernant les mesures présentées dans ce travail.

\section{Conclusion:-}

Le système d'irrigation par pompage photovoltaïque que nous avons monté à des fins d'automatisation et d'économie. En effet, après une étape de vérification de l'efficience des cartes de commande dans un logiciel de simulation, ce système a pu se voir édifié sur une maquette, soit un banc de test qui a témoigné de la réussite des mécanismes de pompage et d'irrigation.

En matière de puisage, la pompe immergée s'est avérée effectivement protégée des fonctionnements à sec, le bassin automatiquement rempli, le débit aussi bien que la consommation hydrique instantanément mesurés et stockés sur PC, les états de la station de pompage visualisés sur un afficheur LCD.

En matière d'irrigation, la pompe surfacique s'est avéré assurer l'arrosage automatique de deux terrains ou plus via les électrovannes et grâce aux capteurs d'humidité. Les risques d'anomalies ont d'autre part fait objet de prévention et d'anéantissement par substitution d'une vanne manuelle à l'une des électrovannes et par recours à un algorithme différent de celui des deux terrains irrigués exclusivement par électrovannes.

En somme, comme valeurs ajoutées, par un tel projet, le fermier se verra réaliser plus de gain par moins de dépenses physiques, énergétiques et financières, l'environnement mieux portant avec des dépenses hydriques rationnalisées et une pollution réduite.

\section{Référence:-}

1. S.S. Chandel, M. Nagaraju Naika, Rahul Chandel,"Review of solar photovoltaic water pumping system technology for irrigation and community drinking water supplies", Renewable and Sustainable Energy Reviews, Elsevier, Vol. 49, pp. 1084-1099, 2015.

2. N.L. Panwara,S.C. Kaushikb,Surendra Kotharia, "Role of renewable energy sources in environmental protection: A review", Renewable and Sustainable Energy Reviews, Elsevier, Vol: 15, Issue 3, pp. 1513-1524, 2011.

3. B. Bouzidi, A. Maleket M. Haddadi,"Rentabilité économique des systèmes de pompage photovoltaïques", Revue des Energies Renouvelables Vol. 9 N³,pp. 187-197, 2006.

4. B. Bouzidi, M. Haddadi, A. Malek, Analyse technico économique comparative de différentes configurations de systèmes de pompage. Revue des Energies Renouvelables CER'07 Oujda, vol. 7, p. 117-120, 2007.

5. B. Chikh-Bled, B. Benyoucef, F. Benyarou, I. Sari-Ali, "Etude technico-économique d'un système de pompage photovoltaïque appliquée à un site saharien", Rev. Energ. Ren.,ICPWE,pp. 61-65, 2003. 
6. Mahjoubi Ammar, Mechlouch Ridha Fethi, Ben Brahim Ammar, "Dimensionnement d'une installation solaire de pompage d'eau pour l'irrigation goutte à goutte au climat de Médenine, Tunisie", Revue des Régions Arides, Numéro 24, pp. 1037-1044, 2009

7. A. Hadj Arab, M. Benghanem, A. Gharbi, "Dimensionnement de Systèmes de Pompage Photovoltaïque", Rev. Energ. Ren. Vol. 8,pp. 19-26, 2005.

8. A. Hadj Arab, F. Chenlo, K. Mukadam, J.L. Balenzategui, "Performance of PV Water Pumping Systems", Renewable Energy, Vol. 18, N², pp. 191-204, 1999.

9. Mahmoud M. Elkholy, Ahmed Fathy, "Optimization of a PV fed water pumping system without storage based on teaching-learning-based optimization algorithm and artificial neural network", Solar Energy, Elsevier, Vol. 139, 1, pp. 199-212, 2016.

10. Yasin Kabalci, Ersan Kabalci, Ridvan Canbaz, Ayberk Calpbinici, "Design and implementation of a solar plant and irrigation system with remote monitoring and remote control infrastructures", Solar Energy, Elsevier, Vol. 139, pp. 506-517, 2016.

11. N. Davies, T.D. Short, A. Hassan, "Optimizing Photovoltaic Water Pumping Systems for Developing Countries Through the Addition of a Novel Induced-flow Subsystem", In Renewable Energy in the Service of Mankind, Vol. II,Springer International Publishing, pp. 483-495, 2016.

12. S. Dasberg, D. Or, "Drip Irrigation", Springer Berlin Heidelberg, 1999.

13. http://www.gotronic.fr/pj2-hc-sr04-utilisation-avec-picaxe-1343.pdf

14. "Flow sensor, YF-DN50," [Online]. Available: http://m-radiokomi.ru/yf-dn50-g2-inch-water-flow-metersensor-flowmeter-caudalimetr-counter-indicator-water-flow-system-gauge-device-10-2001-min/.

15. Site officiel de la plateforme Arduino : www.aduino.cc.

16. https://www.labcenter.com/downloads/\#professional. 